Acta Cryst. (2002). A58 (Supplement), C184

SYNTHESIS OF MESOPOROUS SILICA UNDER WEAK ACIDIC CONDITIONS WITH PLURONIC 123 SURFACTANT

A. Shmakov A. Derevyankin M. Mel'gunov V. Fenelonov V. Romannikov Boreskov Institute for Catalysis SD RAS Pr.Acad.Lavrentieva, 5 NOVOSIBIRSK 630090 RUSSIA

The evaluation of the influence of three most important parameters of synthesis on the structure-texture properties of mesostructured silica, prepared using PLURONIC P123 as a surfactant under weak acidic conditions is reported. These most important parameters are: (1) mixing time, (2) conditions of hydrothermal treatment, and (3) final mixture $\mathrm{pH}$. Aqueous solutions of sodium silicate and hydrochloric acid were used as starting reagents for the syntheses. The results show that both structure and texture properties of mesostructured silica may be varied widely using these parameters. Two most interesting types of mesostructured silica were revealed as a result of this work. The first one includes the systems prepared at relatively short mixing times followed by hydrothermal treatment at relatively low temperatures. The texture of these systems may be described as three dimensional, since it consists of primary (large) and complementary (small and middle) mesopores. This type of systems are very promising for using as templates for synthesys of various derivatives such as mesostructured carbon (e.g., CMK-3). The second one represents the systems prepared at relatively long mixing times and at the mixture $\mathrm{pH}$ range of about 3-3.5 followed by hydrothermal treatment at appropriate temperatures. These systems exhibit: (a) highly ordered hexagonal mesostructure of uniformly sized mesopores (without any complementary ones); (b) negligible lattice contraction during calcination, and (c) high stability of the mesostructure relative to additional treatments of calcined forms. This type of systems has to be considered as very promising basis for synthesis of mesostructured element-silicate heterogeneous catalysts.

Keywords: MESOPHASE MESOPOROUS MATERIALS
Acta Cryst. (2002). A58 (Supplement), C184

\section{ADVANTAGES AND CHARACTERISTICS OF TWO-DIMENSIONAL POWDER DIFFRACTION}

Bob B. He Uwe Preckwinkel Kingsley L. Smith

Bruker AXS 5465 East Cheryl Parkway MADISON WI 53711-5373 USA

In the field of $\mathrm{x}$-ray powder diffraction, data collection and analysis have been based mainly on one-dimensional diffraction profiles measured with scanning point detectors or one-dimensional position-sensitive detectors. In recent years, usage of two-dimensional (2-D) detectors has increased due to the advances in detector technology, x-ray optics, and computing power. The two-dimensional diffraction $\left(\mathrm{XRD}^{2}\right)$ provides far more information than the conventional onedimensional powder diffraction. However, the advantages of a 2-D detector cannot be fully taken if the data interpretation and analysis methods are simply inherited from the conventional diffraction theory. New approaches are necessary to configure the $\mathrm{XRD}^{2}$ system and to analyze the 2-D diffraction data. The presentation discusses some fundamentals about $\mathrm{XRD}^{2}$, including geometry convention, diffraction data interpretation and process, point beam optics, and some application examples. While most conventional diffractometers use the Bragg-Brentano parafocusing geometry in powder diffraction, 2-D diffractometers measure diffraction pattern in a 2-D range with a totally different geometry. In a conventional diffractometer, both the incident $\mathrm{X}$-ray beam and diffracted X-rays can be conditioned before reaching a point detector. However, it is only possible to condition the incident beam in a 2-D diffractometer. Therefore, 2-D diffractometers have different requirements in terms of the beam spectrum purity, divergency and beam cross-section profile. The presentation also introduces an $\mathrm{XRD}^{2}$ system for high-throughput screening.

Keywords: POWDER DIFFRACTION, 2D DETECTOR, HIGHTHROUGHPUT SCREENING
Acta Cryst. (2002). A58 (Supplement), C184

AUTOMATED CRYSTALLOGRAPHIC PHASE IDENTIFICATION USING ELECTRON BACKSCATTER DIFFRACTION D.J. Dingley $1,2,3$

${ }^{1}$ TexSEM Laboratories 392 East 12300 South DRAPER UTAH 84020 USA

${ }^{2}$ EDAX International ${ }^{3}$ University of Bristol

High-resolution high dynamic range charge couple device cameras have been used to record electron backscatter diffraction patterns (EBSPs) from matrix and precipitated phases in metallurgical and mineralogical samples. The patterns are collected in a scanning electron microscope simultaneously with chemical data using energy dispersive spectrometry. Automated diffraction band detection allows measurements of interplanar angles with a precision of $0.2^{\circ}$ and interplanar spacing precision of up to $2 \%$. A typical EBSP contains 30 or more diffraction bands so that there are numerous interband angle and lattice spacing measurements available for comparison with corresponding values calculated from crystal phase data listed in international crystallographic tables. In practice the chemical composition of the phase was used to search the ICDD powder diffraction files, PDF, to extract all recorded phases with that composition. A table of interband angles and lattice spacings was computed for each candidate phase and compared with corresponding measured values. The candidate phase which provided the best match of calculated to observed values was selected and a computer simulation of diffraction band positions for the selected phase overdrawn on the EBSD image so that a visual check could be made. For binary and some ternary compounds the pdf data was applicable to EBSP. However, for more complex compounds there was often only a poor correspondence indicating that in such cases atomic disorder in the compounds was affecting both the scattering factors and point group symmetries.

Keywords: PHASE IDENTGIFICATION, ELECTRON DIFFRACTION, ON-LINE MEASUREMENT

Acta Cryst. (2002). A58 (Supplement), C184

\section{STRUCTURE STUDY OF HYDRATED Zr(1\% Nb) REACTOR FUEL} TUBE CLADDING MATERIAL

$\underline{\text { E. Svab }}^{1}$ Gy. Meszaros ${ }^{1}$ Z. Somogyvari ${ }^{1}$ M. Balasko

${ }^{1}$ Research Institute for Solid State Physics And Optics Konkoly Thege St 3933 BUDAPEST 1121 HUNGARY ${ }^{2}$ Atomic Energy Research Institute

The presence of hydrogen in cladding materials of nuclear fuel pressure tube systems of nuclear plants can lead to hydride induced embrittlement, and also may induce a swelling when they dissolve in the metal matrix. The hydrides may lead to delayed hydride cracking or formation of hydride blisters.

The aim of our work was to analyze hydrated $\mathrm{Zr}-1$ at $\% \mathrm{Nb}$ material - serving as reactor fuel cladding tube in the VVR-SM type reactors - in the $\mathrm{H}$ concentration range of $500-13300 \mathrm{ppm}$. The samples were hydrated in streaming gas in a high temperature furnace of about $1000 \mathrm{C}$. For detection of the H-content and distribution neutron radiography (NR), while for studying the formation of different phases neutron diffraction (ND) was used.

The ND spectra were analyzed by Rietveld method. The patterns of the pure $\mathrm{Zr}-1 \% \mathrm{Nb}$ specimens could be described well in terms of hexagonal $\alpha-\mathrm{Zr}$ $\left(P 6_{3} / m m c\right)$, while the existence of other phases is evident for the hydrated samples. The transformation of $\alpha-\mathrm{Zr}$ into cubic $\delta-\mathrm{ZrH}_{2} \quad(F m-3 m)$ with increasing hydrogen content could be observed, while a small amount of tetragonal $\gamma-\mathrm{ZrH}\left(\mathrm{P}_{2} / n\right)$ was also detected for each hydrated sample. Large texture effects were observed influencing the Bragg intensities and leading to difficulties when performing an accurate quantitative phase analysis. The preferred orientation was analyzed by imaging plate technique.

Keywords: NEUTRON DIFFRACTION, Zr-HYDRIDE, IMAGING PLATE 